\title{
Advances in inborn errors of metabolism
}

\author{
Toshiyuki Fukao ${ }^{1} \cdot$ Kimitoshi Nakamura ${ }^{2}$
}

Received: 31 October 2018 / Accepted: 1 November 2018 / Published online: 25 January 2019

(c) The Author(s) under exclusive licence to The Japan Society of Human Genetics 2018

Inborn errors of metabolism are rare diseases caused by defects of single genes, which code for enzymes that facilitate the conversion of substrates into metabolites. These diseases often arise due to the accumulation of toxic substrates or a deficiency of essential metabolites. The management of inborn errors of metabolism has traditionally consisted in diet therapy and supportive therapy, but recently other treatment options have become available, including enzyme and coenzyme replacement, removal of harmful substances, cell and organ transplantation, and gene therapy. Inborn errors of metabolism are currently one of the hottest topics in medicine since patients with certain inherited metabolic diseases can now be expected to have a good prognosis with appropriate management. Early diagnosis of inborn errors of metabolism is a prerequisite for effective treatment, and treatment optimization is also dependent on accurate prediction of severity and prognosis. Neonatal screening plays an important role in early diagnosis, and use of tandem mass spectrometry has increased the number of diseases that can be detected. Genetic analysis is indispensable for definitive diagnosis. On the basis of genetic analysis, it is possible to predict time of onset and complications, and to initiate the necessary treatment at the optimal time point. However, confirmation of diagnosis by genetic analysis is subject to limitations in terms of cost, time, efficiency, etc., and ethical considerations must also be taken into consideration. It is important to carry out genetic analysis when conducting genetic counseling.

In this issue, eight review articles present the latest knowledge on inborn errors of metabolism. Yamada et al. describe mitochondrial fatty acid oxidation disorders, focusing on the management and diagnosis of very-longchain acyl-CoA dehydrogenase deficiency. Kobayashi et al. introduce trends in mucopolysaccharidosis research. Ohashi discusses gene therapy for lysosomal storage diseases and peroxisomal disorders. Murayama et al. described the diagnosis, molecular genesis, and treatment of mitochondrial disease. Shimozawa et al. report on the use of an efficient diagnostic system for peroxisomal disorders in Japan. Fukao et al. describe advances in the understanding of beta-ketothiolase (mitochondrial acetoacetyl-CoA thiolase, T2) deficiency. Kure and Shintaku introduce tetrahydrobipterin-responsive phenylalanine hydroxylase deficiency. Finally, Tajima et al. describe carnitine palmitoyltransferase II deficiency, with a focus on newborn screening. These articles will clarify the recent progress in understanding of inborn errors of metabolism, their diagnosis, and their management.
Kimitoshi Nakamura

nakamura@kumamoto-u.ac.jp

1 Graduate School of Medicine, Gifu University, Department of Pediatrics, Gifu, Japan

2 Kumamoto University, Department of Pediatrics, Kumamoto, Japan 